Recibido 05 de agosto 2021 - Aceptado 01 de setiembre, 2021

\title{
Pasión y actos delictivos en las barras bravas de fútbol
}

\section{Passion and criminal acts in the football braves}

\author{
Raysa Truyenque 1
}

Felipa Muñoz 2

\section{RESUMEN}

El presente artículo de revisión tiene como título "Pasión y actos delictivos en las barras bravas de fútbol”. Objetivo: Comparar las propuestas sobre los actos delictivos que originan violencia en las barras bravas de fútbol. Método: Estudios descriptivos, de revisión de artículos de científicos en revistas indexadas publicadas en diferentes bases de datos entre los años 2014-2021. Las investigaciones analizadas desarrollaron estudios descriptivos, correlacionales, inductivos. Resultados: Se identificaron 28 artículos relacionados al tema de los cuales se seleccionaron 10. La revisión ha evidenciado que el problema de las barras bravas nace a partir de las rivalidades que presentan los grupos por defender al club de sus amores, siendo un problema social que está relacionado con el fenómeno de las pandillas conformadas por aproximado de 15 y 100 integrantes, provocando inseguridad a determinadas zonas. Conclusiones: La violencia de las barras bravas forma como parte de una característica fundamental, donde manifiestan su lucha, dolor y el riesgo de enfrentarse frente al equipo contrario.

Palabras clave: Violencia, Barras, Fútbol, Hinchadas, Cánticos.

\section{ABSTRACT}

This review article is entitled "Passion and criminal acts in the football braves". Objective: Compare the proposals on the criminal acts that cause violence in the soccer brave bars. Method: Descriptive studies, reviewing articles by scientists in indexed journals published in different databases between the years 2015-2021. The researchers analyzed developed descriptive, correlational, inductive studies. Results: 28 articles related to the subject were identified, of which 10 were selected. The review has shown that the problem of the brave bars arises from the rivalries that the groups present to defend the club of their loves, being a social problem that It is related to the phenomenon of gangs made up of approximately 15 and 100 members, causing insecurity in certain areas. Conclusions: The violence of the brave bars is part of a fundamental characteristic, where they express their struggle, pain and the risk of facing the opposing team.

keywords: Violence, Bars, Soccer, Swollen, Chants.

1. 12vo ciclo de la carrera de Derecho y Ciencias Políticas de la Universidad César Vallejo. Correo electrónico : truyenque16@ucvvirtual. edu.pe - raisatruyenqueq@gmail.com

2. Doctora en Derecho, Investigadora RENACYT. Docente de la Universidad César Vallejo.

w_munozcc@hotmail.com - fmunozcc@ucvvirtual.edu.pe 


\section{INTRODUCCIÓN}

En referencia a la aproximación temática, el presente artículo de revisión abarcó el estudio de esta problemática donde el deporte se ha visto involucrado en temas de violencia originados por los barristas que acuden a los espectáculos deportivos. En la actualidad la violencia de las barras bravas ha sido un problema que no se ha podido resolver, pese a existir un marco jurídico que sanciona los actos vandálicos que origina el ser humano. Aunado a ello, el fútbol representa la alegría de la sociedad, más que cualquier otro deporte, sea el vóley, básquet o natación. De tal manera que los diferentes medios de comunicación dedican los titulares o noticias a través de revistas o periódicos siempre que se juegue un clásico deportivo. Es por ello, que esta problemática debe ser resuelta considerando propuestas y medidas drásticas estudiadas por otros investigadores con la finalidad de erradicar la violencia originada por las barras bravas del fútbol.

Castro (2020), con relación a las barras bravas sostiene que la fidelidad de los hinchas está reflejado a través de las canciones que elaboran con la finalidad de transmitirle ánimos al club. Es importante mencionar que los barristas elaboran cantos a través de burlas, insultos, desafíos y amenazas a sus rivales futbolísticos.

Por lo tal, es importante plantear una interpretación sobre la forma de exponer el cuerpo entre los hinchas que forman una barra brava en Bogotá.

Asimismo, los autores Acuña \& Acuña (2018) sostienen que la violencia verbal es un tipo común de violencia seguida por la simbólica y luego por la física. Aunado a ello, es preciso mencionar que se vulnera el derecho a la integridad de los espectadores, ya que las humillaciones o expresiones afecta y menosprecia el honor de una persona.

De tal manera, Uribe (2019), manifiesta que la violencia social genera perjuicios a los derechos de los ciudadanos, como es el daño a la propiedad privada y las muertes, pese a existir sanciones y leyes que castigan este tipo de actos vandálicos no han logrado evitar que se siga vulnerado la integridad física y psicológica de los espectadores que asisten a un espectáculo deportivo de fútbol. Es por ello que, Preciado (2018), conceptualiza al barrista como gente ociosa, que no trabaja y que solo vive de ser hincha de un equipo, lo cual se cataloga como una fuerza militar que siempre está disponible para alentar al club cada vez que juegue con un rival.

Finalmente, Pérez (2015) sostiene que las barras han tomado características violentas y delictivas por el consumo de drogas y alcohol, siendo una preocupación generada para la sociedad si es no se toman medidas adecuadas para resolver este tipo de problemática. Por ello, que una solución factible seria implementar políticas públicas a fin de erradicar la violencia de las barras bravas.

\section{MATERIALES Y MÉTODOS}

En cuanto al método, para la selección de artículos se emplearon las siguientes bases de datos Ebsco, Dialnet, Scielo, Scopus, ScienceDirect y Web of Science, en español y en inglés lo cual coadyuvó a reforzar este artículo de revisión.

A continuación, se detalla la planificación, búsqueda, organización y análisis de la información:

En la planificación se estableció el objetivo el cual fue comparar las propuestas sobre los actos delictivos que originan violencia en las barras bravas de fútbol, los objetivos de los diferentes artículos de revisión por los autores, luego con la utilización de diferentes términos como violencia, barras, fútbol, hinchadas y cánticos que se trabajaron para las ecuaciones de búsqueda, en base a un periodo de tiempo de los últimos seis años (2015-2021).

Respecto a la búsqueda y captación de información se consideró las palabras claves y el tiempo programado para recolectar la información y se prosiguió a descargar los artículos para que nos faciliten el avance. 
Con relación a la organización y depuración de la investigación, es decir la inclusión de información que tuvo como criterio el objetivo de la revisión. El análisis de la información parte del

Tabla 1: Matriz de Revistas Indexadas desarrollo de las síntesis de la información que permitió identificar los tópicos que aportan al presente trabajo.

\begin{tabular}{|c|c|c|c|c|c|}
\hline $\begin{array}{l}\text { N.º de } \\
\text { búsqueda }\end{array}$ & Autores & $\begin{array}{l}\text { Años de } \\
\text { publicación }\end{array}$ & Títulos & Revistas & $\begin{array}{l}\text { Base de } \\
\text { datos }\end{array}$ \\
\hline 1 & John Castro & 2020 & $\begin{array}{l}\text { Cuerpos con aguante: festividad } \\
\text { y violencia en una barra brava de } \\
\text { Bogotá }\end{array}$ & $\begin{array}{c}\text { Revista de Colombia de } \\
\text { Sociología }\end{array}$ & Scielo \\
\hline 2 & $\begin{array}{l}\text { Diego Londoño, Juan } \\
\text { Arboleda y Gabriel } \\
\text { Prosser }\end{array}$ & 2020 & $\begin{array}{l}\text { Violence from the point of view } \\
\text { of the spectator: Habitus of the } \\
\text { amateur, the fan and the } \\
\text { supporter }\end{array}$ & $\begin{array}{c}\text { Revista Quaderns de } \\
\text { Psicologia. }\end{array}$ & Scopus \\
\hline 3 & Nicolás Uribe & 2019 & $\begin{array}{l}\text { Violence psychology of masses } \\
\text { and soccer bars }\end{array}$ & Revista Criminalidad & Ebsco \\
\hline 4 & Acuña y Acuña & 2018 & $\begin{array}{l}\text { Violence psychology of masses } \\
\text { and soccer bars } \\
\text { Manifestaciones de violencia en } \\
\text { el estadio de fútbol: El caso del } \\
\text { Granada Club de Fútbol. }\end{array}$ & $\begin{array}{c}\text { Revista. Cultura, Ciencia y } \\
\text { Deporte }\end{array}$ & Ebsco \\
\hline 5 & Santiago Preciado & 2018 & $\begin{array}{l}\text { Las barras de fútbol y el poder } \\
\text { político en Colombia }\end{array}$ & Revista Forum & Dialnet \\
\hline 6 & $\begin{array}{c}\text { Nataly Higuita y Jaime } \\
\text { Restreto }\end{array}$ & 2017 & $\begin{array}{c}\text { Construcción de identidad en } \\
\text { jóvenes de Manizales }\end{array}$ & Revista Ánfora & Ebsco \\
\hline 7 & Patricia Salvador & 2016 & $\begin{array}{l}\text { Barras Bravas en Ecuador. } \\
\text { Estudio Iconológico de las } \\
\text { Subculturas del Fútbol }\end{array}$ & $\begin{array}{c}\text { Revista de divulgación } \\
\text { científica de la } \\
\text { Universidad Tecnológica } \\
\text { Idoamérica }\end{array}$ & Dialnet \\
\hline 8 & Valeria Pérez & 2015 & $\begin{array}{l}\text { ESTUDIO DE LA IDENTIDAD } \\
\text { GRUPAL DE LA BARRA BRAVA } \\
\text { “LA GLORIOSA ULTRA SUR 34” } \\
\text { DEL CLUB THE STRONGEST }\end{array}$ & $\begin{array}{c}\text { Revista de Investigación } \\
\text { Psicológica }\end{array}$ & Scielo \\
\hline 9 & $\begin{array}{l}\text { Luisa Miranda, Vanessa } \\
\text { Orrego \& Diana Vera }\end{array}$ & 2015 & $\begin{array}{l}\text { Barra brava, culture, violence } \\
\text { and society: the world of soccer } \\
\text { fans as representation }\end{array}$ & $\begin{array}{c}\text { Revista de Trabajo Social } \\
\text { e Intervención Social }\end{array}$ & Dialnet \\
\hline 10 & $\begin{array}{l}\text { Nicolas Uribe y } \\
\text { Guillermo Castaño }\end{array}$ & 2014 & $\begin{array}{c}\text { Barras de fútbol, consumo de } \\
\text { drogas y violencia. }\end{array}$ & $\begin{array}{c}\text { Revista psicología desde } \\
\text { el Caribe }\end{array}$ & Scielo \\
\hline
\end{tabular}




\section{RESULTADOS}

En el presente artículo de revisión se identificaron 28 artículos relacionados al tema de los cuales se seleccionaron 10. La revisión ha evidenciado que el problema de las barras bravas nace a partir de las rivalidades que presentan los grupos por defender al club de sus amores, siendo un problema social que está relacionado con el fenómeno de las pandillas conformadas por aproximado de 15 y 100 integrantes, provocando inseguridad a determinadas zonas. En la revisión de las revistas se pudo observar que los autores, consideran que las barras bravas muestran comportamientos violentos, lo cual ha generado que la sociedad tenga miedo de asistir a un estadio a disfrutar el fútbol, y a vivir en un ambiente de paz social, siendo un problema que se originan vías públicas donde estos movimientos de grupos causan disturbios y vulneran el derecho de las personas.

Los autores asumen una postura positiva en cuanto a considerar que las barras bravas son fenómenos sociales que se mueven por determinadas zonas para alentar a su club. Tal como lo indica Castro (2020) en los resultados de su investigación se evidenció los diferentes tipos de barristas, por un lado, viajan de manera organizada a través de buses, aviones o cualquier otro transporte público y con las entradas adquiridas para ingresar al estadio, y el otro grupo son los barristas que se movilizan a pie, siendo conocidos como los "caminantes".

A su vez, Londoño, Arboleda y Porsser (2020) en sus resultados construyeron una categoría general: Habitus espectador, en relación a 3 sub categorías habitus aficionado, habitus hincha y habitus barrista, conceptualizando al H. Espectador como aquel sujeto que asiste forma directa a través de su asistencia al estadio. En esa misma línea, el habitus aficionado, no es barrista sino un simpatizante por el fútbol, lo cual no implica alentar ni pelar con el rival contrario, a diferencia de un hincha que presenta rivalidad con el bando contrario y por último el habitus barrista quien tiene un compromiso por seguir al equipo y el consumo de alcohol y drogas como parte de un ritual barrístico. Los autores señalan que los habitus de incorporan a través de ciertas características que aficionan al barrista.

Respecto a la conformación de una barra brava, los autores Uribe \& Castaño (2014), sus resultados fueron la realizaron cinco categorías, como primera categoría, en su gran mayoría los que pertenecen a una barra son hombres que rigen entre los 14 y 35 años, como segunda, son las sustancias toxicas que consume el barrista antes y después del partido, como son la marihuana, el alcohol, el tabaco y por último la cocaína, la tercera categoría es la conducta violenta denominada "pogo" que consiste en golpear y empujarse durante y después de los partidos, la cuarta categoría es el lenguaje grotesco y obsceno que emplean los barristas para dirigirse a los jugadores o técnicos cuando el resultado del partido no es exitoso, y por último la quinta categoría son las conductas de apoyo al equipo, como es quitarse el polo, saltar, aplaudir, hacer ola y entonar los canticos.

Es menester señalar que los autores Acuña y Acuña (2018), en sus resultados señalaron que la violencia verbal es una de las violencias más comunes que se dan en los estadios de Los Cármenes, seguida por la violencia simbólica y por último la violencia física.

En este sentido Uribe (2019) en el resultado de sus investigaciones realizadas en Colombia, uno de los principales factores de violencia en los barristas radican en la imitación del contagio de comportamiento y actitudes de los grupos de barras. Asimismo, con respecto a la actuación violenta e imitaciones no existe una relación de causalidad directa o indirecta entre los fenómenos de la de la psicología de los grupos y el comportamiento violento observado a los barristas, siendo una problemática descrita en reportajes, periódicos e investigaciones científicas. Es por ello que es necesario realizar nuevas investigaciones con la finalidad de demostrar la relación de causalidad a través de dueños metodológicos. 
Por su parte los autores, Miranda, Urrego y Vera (2015), en sus resultados encontraron dos posturas distintas: En la primera resalta que la sociedad cataloga al barrista con calificativos de delincuente, drogadicto, vándalo y desadaptado. La segunda se destaca las manifestaciones artísticas que la barra utilizar para alentar a su equipo. Asimismo, en las investigaciones realizadas, los resultados demostraron que existe siete tipos de barristas: el social, el líder, el académico, el casual, el anímico, el de barra brava y el violento. En su gran mayoría los barristas rigen 10 y 14 años que estarían participando constantemente en la integrando una barra brava, la cual gira en torno para alentar a su equipo como también participar en los enfrentamientos violentos y actos delictivos. En ello también influiría la ocupación del integrante para la construcción de su identidad.

Por su parte, Higuita y Restreto (2017), en sus resultados obtenidos, señalaron que las barras tienen características parecidas a las "tribus urbanas” o "culturas urbanas". En ello, los jóvenes se ven influenciados por ciertas características del contexto agradable a sus convicciones y subjetividades. De igual manera, se demostraron otras particularidades relacionadas a la identidad de los jóvenes en relación a los rituales que tienen las barras bravas, ligadas a la identidad de los jóvenes en relación con los rituales que adquieren en dicha agrupación.

A su vez, Preciado (2017) en el resultado de su investigación identificaron y caracterizaron las expresiones de relación entre las barras de Colombia con el poder político. Entre las principales expresiones de esa relación encontraron la participación comunitaria a través del barrismo social, la creación y la normatividad que regula la participación institucional y el enfrentamiento violento. Asimismo, los comienzos de las barras nacen en Bogotá a partir de los años 1197, 1998 y 1999, con la creación de los equipos de los Comandos Azules, el Barón Rojo y en Medellín Los del Sur. Sin embargo, quien goza con mayor popularidad es el club Atlético Nacional, integrada por un aproximado de 2.000 personas y en días de partidos 10.000, sin exigencia de dinero o requisitos. Es importante mencionar que el liderazgo del barrista comienza a partir de la antigüedad y el reconocimiento del trabajo realizado.

La Dra. Pérez (2015) en sus resultados obtenidos trabajó con dos cuadros A Y B, la aplicación de los instrumentos como la aplicación de cuestionarios y las entrevistas individuales ayudaron a recolectar una gran cantidad de datos para la descripción de la identidad grupal que se dan entre los barristas del club Gloriosa Ultra Sur simpatizante del Club The Strongest de La Paz Bolivia. En el cuadro A, mediante la aplicación de cuestionario, señalaron las expresiones verbales se desarrollan en el Stadium Hernando Siles. Mientras que en el cuadro B Las conductas han sido observadas en toda ocasión, donde se contactó con la población, en ello los gestos que toman los hinchas con la barra contraria e incluso con la policía son características que forman parte de un barrista, siendo posturas determinadas como el aliento siendo una postura para enfrentar al rival en la cancha de fútbol.

Por último, Salvador (2016), en sus resultados de la producción gráfica hallada en el Barcelona Sporting Club, los colores predominantes son el amarillo y negro -colores con los que la institución deportiva maneja su corporatividad. Las imágenes que con mayor frecuencia se pueden encontrar son: el toro o el Minotauro, en varios niveles icónicos desde los más realistas hasta abstracciones requeridas por la composición visual; utilización de elementos relacionados como el puño levantado, la imagen del Che Guevara, de Eloy Alfaro, la palabra revolución, ídolo, sur oscura y elementos de la cultura rastafari. 
Tabla 2: Matriz de Resultado

\begin{tabular}{|c|c|c|c|c|}
\hline Autor & Grupo de Población & Objetivo & Técnica e Instrumento & Resultados \\
\hline John Castro & $\begin{array}{c}\text { Miembros de una barra } \\
\text { brava de Bogotá }\end{array}$ & $\begin{array}{c}\text { Explicar las formas de } \\
\text { exponer el cuerpo entre } \\
\text { los hinchas que integran } \\
\text { una barra brava de } \\
\text { Bogotá }\end{array}$ & $\begin{array}{c}\text { Técnicas } \\
\text { conversacionales, } \\
\text { como la entrevista } \\
\text { semiestructurada }\end{array}$ & $\begin{array}{l}\text { Se ha planteado dos formas de } \\
\text { exponer el cuerpo entre los } \\
\text { hinchas que integran las barras } \\
\text { bravas, a través su } \\
\text { participación en las } \\
\text { expresiones festivas y en los } \\
\text { comportamientos violentos, } \\
\text { manifestaciones y conductas } \\
\text { que son mediadas por el } \\
\text { insulto. }\end{array}$ \\
\hline $\begin{array}{l}\text { Diego Londoño, } \\
\text { Juan Arboleda y } \\
\text { Gabriel Prosser }\end{array}$ & $\begin{array}{l}\text { Se eligieron dos perfiles } \\
\text { de participantes bajo un } \\
\text { muestreo por } \\
\text { conveniencia: de un } \\
\text { lado, sujetos que han } \\
\text { ejercido la violencia } \\
\text { física en el contexto del } \\
\text { fútbol, entrevistando a } 8 \\
\text { participantes. }\end{array}$ & $\begin{array}{l}\text { Analizar las experiencias } \\
\text { vividas y los significados } \\
\text { producidos por personas } \\
\text { que han participado } \\
\text { directa o indirectamente } \\
\text { de las violencias en el } \\
\text { fútbol. }\end{array}$ & $\begin{array}{c}\text { Entrevistas } \\
\text { semiestructuradas: se } \\
\text { hicieron ocho } \\
\text { entrevistas } \\
\text { semiestructuradas con } \\
\text { participantes } \\
\text { involucrados de } \\
\text { manera directa en } \\
\text { hechos de violencia } \\
\text { física o material } \\
\text { asociada al fútbol }\end{array}$ & $\begin{array}{l}\text { Esta investigación hizo especial } \\
\text { énfasis en el rol del espectador } \\
\text { y cómo se configura el habitus } \\
\text { que posibilita la emergencia de } \\
\text { la violencia. Se construyó la } \\
\text { categoría el habitus espectador } \\
\text { de la cual se deprenden tres } \\
\text { subcategorías, a saber, son: } \\
\text { habitus aficionado; habitus } \\
\text { hincha; y habitus barrista. }\end{array}$ \\
\hline Nicolás Uribe & $\begin{array}{l}\text { Grupo de barristas que } \\
\text { pertenecen a una barra } \\
\text { brava. }\end{array}$ & $\begin{array}{l}\text { Realizar una comparación } \\
\text { entre el concepto de } \\
\text { masa psicológica (de la } \\
\text { Psicología Social y el } \\
\text { Psicoanálisis) barras de } \\
\text { fútbol }\end{array}$ & $\begin{array}{l}\text { La herramienta de } \\
\text { recolección de } \\
\text { información fue la } \\
\text { revisión documental, } \\
\text { pues permite tratar los } \\
\text { textos, libros, } \\
\text { reportajes, entre otros } \\
\text { materiales, }\end{array}$ & $\begin{array}{l}\text { Investigaciones realizadas en } \\
\text { Colombia muestran que } \\
\text { algunos de los principales } \\
\text { factores que inciden en el } \\
\text { actuar violento de los sujetos } \\
\text { en las barras de fútbol } \\
\text { radicaban en los fenómenos de } \\
\text { identificación, sugestión e } \\
\text { imitación o contagio de } \\
\text { actitudes y comportamientos. }\end{array}$ \\
\hline
\end{tabular}




\begin{tabular}{|c|c|c|c|c|}
\hline Acuña y Acuña & $\begin{array}{c}\text { Se eligieron a miembros } \\
\text { pertenecientes a las } \\
\text { barras bravas. }\end{array}$ & $\begin{array}{l}\text { El propósito es resaltar } \\
\text { las dinámicas de } \\
\text { cooperación y de } \\
\text { competencia de } \\
\text { consenso y de conflicto. }\end{array}$ & $\begin{array}{c}\text { Técnica empleada } \\
\text { (observación } \\
\text { sistemática, } \\
\text { conversaciones } \\
\text { informales y } \\
\text { entresvistas). }\end{array}$ & $\begin{array}{l}\text { Según el resultado por la } \\
\text { totalidad de las personas } \\
\text { entrevistadas, señalan que la } \\
\text { violencia verbal es el tipo más } \\
\text { común. }\end{array}$ \\
\hline $\begin{array}{l}\text { Santiago } \\
\text { Preciado }\end{array}$ & Grupos de barristas & $\begin{array}{c}\text { El objetivo de este } \\
\text { artículo es caracterizar } \\
\text { las expresiones que se } \\
\text { dan en torno a la relación } \\
\text { entre las barras } \\
\text { populares y el poder } \\
\text { político en Colombia }\end{array}$ & $\begin{array}{c}\text { Recolección y el } \\
\text { análisis de los datos, } \\
\text { así mismo se hace una } \\
\text { revisión bibliográfica, } \\
\text { de prensa y la } \\
\text { realización de } \\
\text { entrevistas a } \\
\text { informantes clave. }\end{array}$ & $\begin{array}{l}\text { Se identificó las expresiones de } \\
\text { relación entre las hinchadas } \\
\text { futbolísticas o barras en } \\
\text { Colombia con el poder político. }\end{array}$ \\
\hline $\begin{array}{l}\text { Nataly Higuita y } \\
\text { Jaime Restreto }\end{array}$ & $\begin{array}{l}\text { Grupo de jóvenes que } \\
\text { pertenecen a una barra } \\
\text { brava }\end{array}$ & $\begin{array}{l}\text { Describir el proceso de } \\
\text { construcción de la } \\
\text { identidad de jóvenes } \\
\text { adscritos a barras de del } \\
\text { fútbol, }\end{array}$ & $\begin{array}{l}\text { Se utilizaron dos } \\
\text { técnicas: 1) Grupo } \\
\text { focal y 2) Entrevistas }\end{array}$ & $\begin{array}{l}\text { Se evidenció un índice alto de } \\
\text { filiación de los jóvenes a las } \\
\text { barras y que la fidelidad y la } \\
\text { violencia son sus principales } \\
\text { características. }\end{array}$ \\
\hline $\begin{array}{l}\text { Patricia } \\
\text { Salvador }\end{array}$ & $\begin{array}{l}\text { Barristas de } 3 \text { equipos de } \\
\text { fútbol ecuatoriano. }\end{array}$ & $\begin{array}{l}\text { Encontrar material visual, } \\
\text { siendo el objetivo de este } \\
\text { texto el análisis del } \\
\text { mismo. }\end{array}$ & $\begin{array}{c}\text { Observación de } \\
\text { estadios, reuniones } \\
\text { con barristas, } \\
\text { entrevistas y grupos } \\
\text { focales, fueron } \\
\text { herramientas } \\
\text { adicionales que } \\
\text { ayudaron a construir la } \\
\text { diégesis en la que se } \\
\text { encuentran los } \\
\text { barristas }\end{array}$ & $\begin{array}{c}\text { Dentro de la producción gráfica } \\
\text { hallada en el Barcelona } \\
\text { Sporting Club, los colores } \\
\text { predominantes son el amarillo } \\
\text { y negro -colores con los que la } \\
\text { institución deportiva maneja su } \\
\text { corporatividad }\end{array}$ \\
\hline
\end{tabular}




\begin{tabular}{|c|c|c|c|c|}
\hline Valeria Pérez & $\begin{array}{c}\text { Integrantes de una barra } \\
\text { brava }\end{array}$ & $\begin{array}{c}\text { Describir la Identidad } \\
\text { Grupal de la Barra Brava } \\
\text { denominada “Gloriosa } \\
\text { Ultra Sur" simpatizante } \\
\text { del Club The Strongest de } \\
\text { La Paz-Bolivia. }\end{array}$ & $\begin{array}{l}\text { En esta investigación se } \\
\text { emplean técnicas e } \\
\text { instrumentos de } \\
\text { Observación. }\end{array}$ & $\begin{array}{l}\text { La información obtenida } \\
\text { gracias a la Observación } \\
\text { Sistemática, la aplicación de } \\
\text { Cuestionarios, el Grupo Focal y } \\
\text { las Entrevistas Individuales a } \\
\text { los representantes de la Barra, } \\
\text { nos brindan gran cantidad de } \\
\text { datos para la descripción de la } \\
\text { identidad grupal q }\end{array}$ \\
\hline $\begin{array}{c}\text { Miranda, } \\
\text { Orrego y Vera }\end{array}$ & $\begin{array}{l}\text { Miembros de una Barra } \\
\text { Brava }\end{array}$ & $\begin{array}{c}\text { Mostrar las } \\
\text { representaciones sociales } \\
\text { que sobre la barra brava } \\
\text { Barón Rojo Sur }\end{array}$ & $\begin{array}{c}\text { Técnicas } \\
\text { conversacionales, } \\
\text { como la entrevista } \\
\text { semiestructurada a } \\
\text { actores comunitarios }\end{array}$ & $\begin{array}{l}\text { Se encontraron dos posturas: } \\
\text { Se cataloga despectivamente al } \\
\text { barrista con calificativos como } \\
\text { delincuente y manifestaciones } \\
\text { artísticas que la barra utiliza } \\
\text { para alentar a su equipo }\end{array}$ \\
\hline $\begin{array}{l}\text { Nicolás Uribe \& } \\
\text { Ignacio Castaño }\end{array}$ & $\begin{array}{c}\text { Grupo de jóvenes } \\
\text { pertenecientes a una } \\
\text { barra brava. }\end{array}$ & $\begin{array}{l}\text { Analizar la coocurrencia } \\
\text { entre el consumo de } \\
\text { sustancias psicoactivas y } \\
\text { los comportamientos } \\
\text { agresivos y violentos en } \\
\text { miembros de algunas de } \\
\text { las barras de fútbol de la } \\
\text { ciudad de Medellín, } \\
\text { Colombia. }\end{array}$ & $\begin{array}{l}\text { La técnica utilizada en } \\
\text { esta investigación fue } \\
\text { la observación } \\
\text { participante, debido a } \\
\text { que permite "captar la } \\
\text { realidad social y } \\
\text { cultural de una } \\
\text { sociedad o grupo social } \\
\text { determinado. }\end{array}$ & $\begin{array}{l}\text { Respecto a la categoría } 1 \text {, los } \\
\text { observadores informaron que } \\
\text { los miembros de estas barras } \\
\text { en su mayoría son hombres en } \\
\text { edades comprendidas entre los } \\
14 \text { y los } 35 \text { años, } \\
\text { aproximadamente. }\end{array}$ \\
\hline
\end{tabular}

\section{DISCUSIÓN}

Respecto a los resultados de los autores mencionados, radican en los comportamientos, características y el desplazamiento de las barras bravas, en ello también la representación del símbolo de la insignia y los colores de cada equipo ha sido un punto primordial para analizar la pasión y el hinchaje que tienen los barristas para alentar a su club. Si bien es cierto, el problema de las barras bravas ha ocasionado que la seguridad ciudadana sea la más afectada debido a la vulneración de los derechos de cada persona, como son el daño a la propiedad, la violencia física, las amenazas y los disturbios que ocasionan los barristas cuando se juega un clásico deportivo.

Asimismo, los autores Castro (2020) y Acuña \& Acuña (2018), concuerdan que la violencia verbal es una de las formas de expresar violencia frente al equipo contrario, es por ello que los cánticos que realizan las hinchadas estas destinadas alentar al equipo y si vez ofender al hincha rival. Apreciación: Es menester señalar que una de 
las características principales del barrista son los términos verbales que utilizan, lo cual tiene como finalidad ofender al rival ya sea a través de los canticos como también a través de las redes sociales.

En ello, Londoño, Arboleda y Porsser (2020) \& los autores Uribe \& Castaño (2014, concuerdan que uno de los factores que hacen que una barra brava presente actitudes violentas, es debido al consumo de sustancias toxicas como son las drogas, el alcohol, el tabaco, la marihuana y la cocaína.

Apreciación: Las sustancias toxicas son un problema que no solo parte de las barras bravas, sino también en las escuelas, universidad, colegios, y en ello los jóvenes recaen en este tipo de drogas por la falta de orientación y apoyo familiar, donde la pobreza es uno de los principales factores a que el ser humano acceda a comercializar y a consumir estas drogas. Es importante mencionar, el olor a la marihuana en las tribunas de los estadios que causa una gran incomodidad para los espectadores que acuden a disfrutar del partido, siendo un problema que no se ha podido resolver.

Mientras que los autores Uribe (2019) y Miranda, Urrego y Vera (2015), Higuita y Restreto (2017) sus resultados concuerdan, mientras que el autor Uribe señala que uno de los principales factores de violencia en los barristas radican en la imitación del contagio de comportamiento y actitudes de los grupos de barras, los autores Miranda, Urrego y Vera, \& Huiguita y Restreto (2017) señalan que uno de los factores de las barras bravas están relacionadas al ritual que tienen los barristas, siendo actitudes y comportamientos que copian en la agrupación, empleando características parecidas a las "Culturas Urbanas".

Apreciación: El ser humano que toma la decisión de formar parte de una barra brava, copia actitudes de otros barristas con la finalidad de formar su propia identidad, en ello está incluido las asistencias partido, el lenguaje verbal que emplean y la vestimenta.
En cuanto a los autores Preciado (2017) y Pérez (2019), ambos han desarrollado diferentes resultados, por parte del autor Preciado señala que el club que goza con mayor popularidad es el club Atlético Nacional con la participación de 10,000 barristas y teniendo como líder de la barra a una persona que tenga antigüedad y reconocimiento del trabajo realizado. Mientras que la autora Pérez, a través del desarrollo de su cuadro A Y B, señala que el aliento es una postura enfrentar al rival en la cancha de fútbol.

Apreciación: Los equipos de fútbol que gozan de mayor popularidad, reciben alientos por parte del apoyo de su gente, en ello el aliento del barrista también sirve para motivar a los jugadores a que ganen el partido.

Por último, Salvador (2016), en sus resultados obtenidos señala la importancia de las figuras y los colores del equipo de fútbol.

Apreciación: El significado de los colores y el símbolo que representa a los equipos, son características que presentan los barristas, por ejemplo, el color de los globos, las banderas, antorchas, las figuras representativas de cada club, significan el apoyo que emplean los barristas para alentar a su equipo.

\section{CONCLUSIONES}

1) Para erradicar este tipo de problemática de las barras bravas es importante que se fomenten políticas públicas, con la finalidad de erradicar la violencia de los barristas.

2) La violencia de las barras bravas forma como parte de una característica fundamental, donde manifiestan su lucha, dolor y el riesgo de enfrentarse frente al equipo contrario.

\section{Recomendaciones}

1) Se recomienda presencia de las fuerzas armadas para erradicar la violencia de las barras bravas, ya que el apoyo policial no es suficiente para evitar las desgracias que ocurren en los espacios 
públicos, donde la propiedad y la vida de las personas son las más afectadas.

\section{REFERENCIAS BIBLIOGRÁFICAS}

1) Castro, J. (2020). Cuerpos con aguante: festividad y violencia en una barra brava de Bogotá. Revista Colombia de Sociología, 43(1), 193-214. Recuperado de: http://www.scielo.org.co/scielo.php?script=sci_arttext\&pi$\mathrm{d}=$ S0120-159X2020000100193

2) Londoño, D. Arboleda, J. \& Prosser G. (2020). Las violencias desde el espectador de fútbol: habitus del aficionado, el hincha y el barrista. Revista Quaderns de Psicologia. 22(3). Recuperado de: file://C:/Users/Raysa\%20TQ/Downloads/15336517-2-PB.pdf

3) Uribe, N. (2019). Violence, psychology of masses and soccer bars. Revista Criminalidad. 61(1), 85-96. Recuperado de: http://www.scielo.org.co/ pdf/crim/v61n1/1794-3108-crim-61-01-00085. pdf

4) Acuña, A. \& Acuña, G. (2018). Manifestaciones de violencia en el estadio de fútbol: El caso de Granada Club de Fútbol. Revista Cultura, Ciencia y Deporte. (13), 231-242. Recuperado de http://eds.a.ebscohost.com/eds/ pdfviewer/pdfviewer?vid=1\&sid=4cf7b3a9-bcfb-4446-a494-bf26301962e2\%40sessionmgr4006

5) Uribe, N. (2019). Violence, psychology of masses and soccer bars. Revista Criminalidad. 61(1), 85-96. Recuperado de http://www.scielo.org.co/ pdf/crim/v61n1/1794-3108-crim-61-01-00085. pdf

6) Preciado, S. (2018). Las barras de fútbol y el poder político en Colombia. Revista Forum. (14), 108-205. Recuperado de

file://C:/Users/Raysa\%20TQ/Downloads/DialnetLasBarrasDeFutbolYElPoderPoliticoEnColombia-6751591\%20(1).pdf
7) Higuita, N. \& Restrepo, J. (2017). Construcción de identidad en jóvenes de Manizales vinculados a barras de fútbol. Revista Ánfora. 24(42), 165 - 187. Recuperado de file://C:/Users/ Raysa\%20TQ/Downloads/169-Texto\%20del\%20 art\%C3\%ADculo-450-1-10-20170620.pdf

8) Salvador, P. (2016). Barras Bravas en Ecuador. Estudio Iconológico de las Subculturas del Fútbol. Revista de divulgación científica de la Universidad Tecnológica Idoamérica. 5(1), 32-49. Recuperado de file://C:/Users/Raysa\%20TQ/ Downloads/Dialnet-BarrasBravasEnEcuadorEstudioIconologicoDeLasSubcul-6163732.pdf

9) Pérez V. (2015). ESTUDIO DE LA IDENTIDAD GRUPAL DE LA BARRAS BRAVA "LA GLORIOSA ULTRA SUR 34" DEL CLUB THE STRONGEST. Revista de Investigacion Psicologica. (14), 43-55. Recuperado de http://www. scielo.org.bo/pdf/rip/n14/n14_a05.pdf

10) Uribe, N. \& Castaño, G. (2014). Barras de fútbol, consumo de drogas y violencia. Revista psicología desde el Caribe. 31(2). Recuperado de http://www.scielo.org.co/pdf/psdc/v31n2/ v31n2a05.pdf 